\title{
A Hedonic Approach to Estimate the Price of Reliability, Energy Efficiency and Safety for New Cars in Switzerland
}

\author{
Francesco Moresino \\ Geneva School of Business Administration (HES-SO), Geneva, Switzerland \\ Email: francesco.moresino@hesge.ch
}

How to cite this paper: Moresino, F. (2019) A Hedonic Approach to Estimate the Price of Reliability, Energy Efficiency and Safety for New Cars in Switzerland. American Journal of Industrial and Business Management, 9, 468-481.

https://doi.org/10.4236/ajibm.2019.93031

Received: February 4, 2019

Accepted: March 11, 2019

Published: March 14, 2019

Copyright $\odot 2019$ by author(s) and Scientific Research Publishing Inc. This work is licensed under the Creative Commons Attribution International License (CC BY 4.0).

http://creativecommons.org/licenses/by/4.0/

\begin{abstract}
When purchasing a car in Switzerland, consumers can consult three quality labels. First, a reliability label provides the probability that a given car will have a breakdown in the next year. Second, an energy efficiency label, based on fuel consumption and carbon dioxide emissions, ranks cars from A to G. Third, a safety label, based on crash tests, ranks cars from five to one stars. The aim of this paper is to establish whether these labels have an impact on the price of new cars in Switzerland. Using a hedonic regression model, we show that the reliability label influences the price. More precisely, a decrease by one per thousand of the breakdown's probability increases, on average, the car price by 639 Swiss francs. For the energy efficiency and the safety labels, the hedonic model attains its limits due to collinearity issues and the question cannot be answered in a satisfactory way.
\end{abstract}

\section{Keywords}

Hedonic Price, Car Reliability, Car Energy Efficiency, Car Safety

\section{Introduction}

The Swiss car market is very interesting as no domestic car manufacturers are present. It provides therefore an appealing benchmark to study the behaviour of consumers without facing a domestic bias. In this small country of about 8 million inhabitants, around 4.5 million cars are registered. Founded in 1896, the Touring Club of Switzerland (TCS) has 1.6 million members and is an inevitable actor in the Swiss automotive sector. Among others, TCS provides useful information for people who want to buy a car. For instance, they have a database containing comprehensive characteristics for the majority of car models availa- 
ble in Switzerland. Furthermore, they also perform independent tests for most of them. These tests are greatly prized by people facing the decision of choosing a new car. They also contribute to disseminating three quality labels that guide consumers in their choice. The first one is a reliability label and is computed by the German automobile club Allgemeiner Deutscher Automobil-Club (ADAC). ADAC offers to more than 18 million members breakdown assistance and, from this, compiles breakdown statistics each year and publishes reliability indices for car models. More precisely, this index is the probability for a car of a given model and a given age to have a breakdown in the next year [1]. As the database is huge, this index is extremely reliable. This label can be seen as the European equivalent of the American Car Reliability index constructed by Consumer Reports [2]. The second label is the energy efficiency label introduced by the Swiss Federal Office of Energy (SFOE) in 2003 that ranks cars from A, the best, to G, the worst. This label is based on fuel consumption and carbon dioxide emissions [3]. The third label is the five-star safety rating system provided, since 1997, by the independent organization European New Car Assessment Program (Euro NCAP). For this label, the safest cars are rated with five stars and the safety decreases with the number of stars. This safety rating is determined from different tests, including crash tests [4].

The aim of this paper is to establish, using a hedonic approach, whether these three quality labels have an impact on the price of new cars. Hedonic pricing supposes that the price of a product can be explained by its characteristics and has become a popular method among researchers. It permits, among other things, estimating the value of each single characteristic of the product, or, in other words, each single attribute. Though Haas [5] seems to have been the first to use a hedonic approach, Court [6] is generally considered as the pioneer of hedonic pricing. In this original paper, probably for the first time, the adjective "hedonic" is used to describe the method. Later, in the 1960s, the advent of the computer permitted the expansion of the method. In that decade, researchers like Griliches [7], with his seminal paper, popularised the method. Soon, hedonic approaches were used in economic models. For instance, [8] proposed a hedonic approach to consumer theory and [9] developed an equilibrium theory based on hedonic prices.

For cars, hedonic price indices have been widely used as they clearly outperform standard indices [6] [7] [10] [11] [12] [13] [14]. Indeed, for a sector where rapid changes of quality arise, standard indices like the Laspeyres or Paasche indices should be used with caution when analysing the evolution of prices.

Besides indices, hedonic pricing has also been extensively used to estimate the value of a car's attributes. For the attributes considered in the present paper, namely, reliability, energy efficiency and safety, we can mention, among others, the following studies.

For the reliability attribute, [15] uses the trouble index from Consumer Reports and shows it has an impact on price. Reference [16] utilises an aggregate 
index based on the reliability ratings from Consumer Reports and establishes that consumers value reliability consistently with the analysis of prospect theory. For used cars, [17] takes, as the reliability attribute, the annual mileage, and finds it has an impact on the price.

For the attribute of energy efficiency, the results are disparate. Reference [18] investigates whether miles per gallon (MPG) have an impact on the price, and obtained mixed results, probably due to collinearity. Using an indirect hedonic procedure in order to avoid collinearity, [19] shows that the demand for fuel efficiency in the US increases as the price of petrol increases. However, a decade later, and in the UK, [20] finds, using the same procedure, that the price of petrol has only a small effect on fuel efficiency. Reference [10] establishes that MPG has an impact on price for 72 percent of the period studied. Reference [21] finds that a car's price takes into account fuel economy in a way suggesting that consumers value fuel cost savings in a rational way. Finally, [22] estimates the effect of SFOE's energy efficiency label on price and, using a regression discontinuity design in order to circumvent collinearity problems, finds that the A label increases the price by 6 to 11 percent.

For the safety attribute, [23] takes the braking distance, the number of safety features included in the standard equipment, and the proportion of models with speed regulation devices, and shows that none has a statistical impact on price. Reference [15] determines that the overall injury index computed by the Highway Loss Data Institute has an impact on price. Reference [24] establishes that both mortality and injury risks have an effect on price. Reference [25] considers the probabilities of a fatal accident and of a non-fatal accident, and shows that the first has an impact on price whereas the second has no statistically significant impact on price.

In the present paper, we establish that the reliability influences the price of new cars in Switzerland. More precisely, a decrease by one per thousand of the breakdown's probability increases, on average, the car price by 639 Swiss francs. The research proposed in this article is original in two ways. To our knowledge, it is the first time that the ADAC reliability label has been used in a hedonic pricing model. The second original contribution is the choice of the sample. Indeed, to avoid overwhelming the regression with many options, we only took into consideration car models with homogeneous optional features.

The present paper is organised as follows. In Section 2, we present the methodology and data used for this study. In Section 3, the findings are presented and discussed. Finally, in Section 4, we conclude and outline further research.

\section{Methodology}

\subsection{Hedonic Pricing}

Hedonic regression is a powerful tool for estimating the revealed value of the salient attributes characterizing a given product or service. Roughly speaking, it consists of explaining the price, the dependent variable, by different salient 
attributes, the predictors, using standard regression techniques. For attributes of a qualitative nature, so-called dummy variables are necessary. The dummy variable is set to 1 if the characteristic is present and 0 otherwise.

For our study, the hedonic regression can be written as

$$
P_{i}=\beta_{0}+\sum_{j} \beta_{j} x_{i j}+\varepsilon_{i},
$$

where $P_{i}$ represents the price of the car model $i, x_{i j}$ the value of the attribute $j$ for the car model $i, \beta_{j}$ the regression coefficient for the attribute $j, \beta_{0}$ the intercept coefficient, and $\varepsilon_{i}$ an error term for the price prediction of the car model $i$. As usual, one seeks the coefficients that minimize the sum of the squared error terms. The regression coefficients are a good estimate of the value of the corresponding attribute. Very often, a semi-logarithmic model is used. In such a model, the price is replaced with $\ln \left(P_{i}\right)$ and the regression coefficients give an estimate of the price elasticity of demand for the corresponding attribute.

The principal difficulty in hedonic regression is the choice of the predictors that will be retained in the model. Obviously, predictors with a strong significance must be incorporated in the model and predictors with a low significance must be discarded. Sometimes, this task is not so easy, as the significance of a predictor depends on the predictors that are already incorporated in the model. The classical way to handle this problem is to use stepwise regression.

\subsection{Stepwise Regression}

Although very controversial, stepwise regression remains a widely used method for selecting predictors in a hedonic regression. At each step, variables are included or excluded in the model considering a given criterion. Usually the criterion chosen is a $t$-test, an $F$-test, or the Akaike information criterion (AIC) proposed by [26] [27]. The three main methods for stepwise regression are forward selection, backward elimination, and the bidirectional approach. In forward selection, the initial model contains no variables. Then, in light of the chosen criterion, each variable is tested and the variable providing the best improvement of the fit is included in the model. The procedure is repeated until no additional inclusion improves the model with statistical significance. Backward elimination starts with a model containing all variables considered. Then, in light of the chosen criterion, the variable with the most insignificant deterioration of the fit is excluded. The procedure is repeated until no variable can be excluded without altering the fit with statistical significance. The bidirectional approach combines both previous methods, to include and exclude variables.

It is beyond the scope of this article to discuss the weakness of stepwise regression (see for example [28] [29] [30]), but we can mention the two main drawbacks. First, stepwise regression will not necessarily produce the best model and, second, the model is not as accurate as the statistics seem to show (inflated $R$-squared, confidence intervals too narrow, etc.).

\subsection{Collinearity}

It is well known that a strong correlation between two or more predictors causes 
problems for multiple regression (see for example [31] [32]). Unfortunately, as many studies have already pointed out, a car's salient attributes can be highly correlated with each other (see for example [7] [18] [19] [23] [33]). The easiest way to bypass this problem is to discard variables that are strongly correlated with other ones. This radical method has however a major drawback: it could exclude variables affecting the price.

A smarter way to handle the problem of collinearity is to use a principal component regression. For this method, first a principal component analysis (PCA) is performed on all the predictors considered [34] [35]. Roughly speaking, a PCA finds orthogonal factors that explain, in an optimal way, the variance of all the predictors considered. Then a regression is performed on these factors (or a subset of them) and, as they are orthogonal, no collinearity is encountered. The selection of the regression's predictors (in this case the factors) can be done exactly in the same way as a standard regression.

\subsection{Data}

For this study, we considered cars' technical data that are easily available to Swiss consumers. As mentioned earlier, the TCS provides a user-friendly website gathering all the information of interest for people wanting to buy a car [36]. As this website offers comprehensive information and is probably the most popular of its kind, we collected data only from this source. Although the ADAC breakdown statistics are available on the TCS website, we collected them from [1].

The predictors considered for the hedonic model are listed in Table 1. Let us recall that we utilise the ADAC breakdown statistics ${ }^{1}$ the SFOE energy efficiency label and the Euro NCAP safety label. Note that in our study, all cars have a safety label better than or equal to three stars.

For the qualitative variables, the modelling was done with so-called dummy variables. For instance, for the drive wheels characteristic, the variable DRIVE can take three different values (front-wheel, rear-wheel and four-wheel) and two dummy variables are necessary for the model. The first dummy variable, FRONT-WHEEL, is set to 1 in the case of a front-wheel drive and 0 otherwise. The second variable, REAR-WHEEL, is set to 1 in the case of a rear-wheel drive and 0 otherwise. The third dummy variable, namely FOUR-WHEEL, is obviously redundant and should not be included in the regression as it could create collinearity problems. Similarly, for each qualitative variable, one dummy variable is redundant and must be discarded.

In Switzerland, as in many other countries, car manufacturers propose, for each model, a basic version that can be personalised by adding optional features. The list of options is considerably long and, if they are taken into account in the model, will necessitate many variables that will overwhelm the hedonic regression. In this study we can avoid this issue by taking into account, for each car ${ }^{1}$ More precisely, as this study considers new cars, we take the statistics for the youngest cars. 
Table 1. Predictors considered.

\begin{tabular}{|c|c|c|}
\hline \multicolumn{3}{|c|}{ Quantitative variables } \\
\hline Name & & Description \\
\hline BREAKDOWN & \multicolumn{2}{|c|}{ Probability to have a breakdown in the next year. This is the reliability factor. } \\
\hline POWER & \multicolumn{2}{|l|}{ Power in PS. } \\
\hline LENGTH & \multicolumn{2}{|l|}{ Length in centimetres. } \\
\hline DOORS & \multicolumn{2}{|l|}{ Number of doors. } \\
\hline PLACES & \multicolumn{2}{|l|}{ Passenger places. } \\
\hline LOAD & \multicolumn{2}{|l|}{ Car load in kilogrammes. } \\
\hline WEIGHT & \multicolumn{2}{|l|}{ Weight in kilogrammes. } \\
\hline FUEL & \multicolumn{2}{|c|}{ Fuel consumption in litres per hundred kilometres. } \\
\hline $\mathrm{CO} 2$ & \multicolumn{2}{|c|}{ Carbon dioxide emissions in grammes per kilometre. } \\
\hline TORQUE & \multicolumn{2}{|c|}{ Engine torque in Newton-meter. } \\
\hline \multicolumn{3}{|c|}{ Qualitative variables } \\
\hline Name & Description & Dummy variables \\
\hline ENERGY & Energy efficiency label & $\mathrm{A}, \mathrm{B}, \mathrm{C}, \mathrm{D}, \mathrm{E}, \mathrm{F}$ and $(\mathrm{G})$ \\
\hline SAFETY & Safety label & 5-STARS, 4-STARS and (3-STARS) \\
\hline ENGINE & Engine position & FRONT-ENGINE and (REAR-ENGINE) \\
\hline DRIVE & Drive wheels & $\begin{array}{l}\text { FRONT-WHEEL, REAR-WHEEL and } \\
\text { (FOUR-WHEEL) }\end{array}$ \\
\hline CLASS & Class according to TCS & SUPERIOR, MEDIUM, LOWER-MEDIUM, \\
\hline & & $\begin{array}{l}\text { CROSS-COUNTRY, MINIVAN, } \\
\text { SMALL and (MICRO) }\end{array}$ \\
\hline BRAND & Brand & AUDI, BMW, CHEVROLET,... \\
\hline
\end{tabular}

The redundant dummy variables are in brackets.

model, only one version: the most basic version with the minimal features given in Table 2.

In Switzerland, the market is dominated by the petrol engine, that accounts for 76 percent of the market, and we therefore did not consider diesel, liquefied petroleum gas (LPG), or electric engines. Though the share of automatic transmission has drastically increased, from 20 to 45 percent in the last two decades, manual transmission still remains more popular. For this study, we therefore consider only the version with manual transmission. Few brands proposed basic models without air conditioning or a global position system (GPS). For these cases, we chose the most basic version having both options. Finally, the six last features in Table 2 were included in the basic version of all models.

To conclude, note that the sample contains 65 distinct models made by 22 different manufacturers and covers the vast majority of cars sold in Switzerland.

\section{Empirical Results}

To select the predictors for the hedonic regression, we used the three standard methods of stepwise regression. For the criterion, we considered two alternatives: 
Table 2. Car features.

Petrol engine
Manual transmission
Air conditioning
Global position system (GPS)
Anti-lock braking system (ABS)
Electronic stability program (ESP)
Power steering
Airbag driver
Airbag passenger
Airbags lateral

the AIC and a two tailed $t$-test. For both criteria, we additionally imposed that variables which are not strongly significant be discarded from the model. For this purpose, we used a two tailed $t$-test and fixed the significance level at 0.01 . The numerical computations were done with the $\mathrm{R}$ software package.

\subsection{The Hedonic Model}

First, we investigated linear regressions considering all predictors. When a problem of collinearity was encountered, the less significant variable was excluded from the model. The different methods lead to the hedonic pricing model

$$
P \approx 258.81 \cdot \text { POWER }-639.14 \cdot \text { BREAKDOWN }+8345.68 \cdot \text { SUPERIOR }
$$

with an adjusted $R$-squared of 0.9797 . Details of the regression can be found in Table 3.

The resulting model has an excellent adjusted $R$-squared (0.9797) with only three predictors. In addition, each of the three predictors has a very good significance (two sided $t$-test $p$-values below $3.15 \cdot 10^{-4}$ ). Note that although the predictor TORQUE has a good $p$-value (0.0018), it has been excluded from the regression due to the high correlation (0.94) with the predictor POWER. Finally, note that although two brands (HYUNDAI respectively MERCEDES) have good $p$-values (0.0079 respectively 0.0040$)$, they have been excluded from the regression due to their dependence on the variable BREAKDOWN. To establish this, we performed a Kruskal-Wallis test and reject the hypothesis that the variables BRAND and BREAKDOWN are independent ( $p$-value $=0.0005)$. We did not use the more classic analysis of variance (ANOVA) as the normality assumption was not met (Shapiro-Wilk test of normality with $p$-value $8.41 \cdot 10^{-5}$ ). Note that ANOVA, even if not used, confirms the Kruskal-Wallis test ( $p$-value $=$ $\left.3.42 \cdot 10^{-9}\right)$. The exclusion of the variable TORQUE is obvious but the exclusion of the variable BRAND is less evident. We therefore also investigated models where this variable was not excluded. The different methods lead to four different hedonic models: a first model containing 10 predictors with an adjusted 
Table 3. Regression results. The price $p$ is the dependent variable.

\begin{tabular}{cccccc}
\hline Predictor & Estimate & Std. Error & $t$ value & $p$-value (two sided) & \\
\hline POWER & 258.81 & 6.84 & 37.84 & $<2 \cdot 10^{-16}$ & $* * *$ \\
BREAKDOWN & -639.14 & 125.71 & -5.08 & $3.66 \cdot 10^{-6}$ & $* * *$ \\
SUPERIOR & 8345.68 & 2186.53 & 3.82 & $3.15 \cdot 10^{-4}$ & $* * *$ \\
\hline
\end{tabular}

${ }^{* *} p$-value $<0.001$ (two sided). Adjusted $R^{2}: 0.9797 . R^{2}: 0.9806$.

$R$-squared of 0.9915 , a second model containing 11 predictors with an adjusted $R$-squared of 0.9908 , a third model containing 12 predictors with an adjusted $R$-squared of 0.9928 , and a fourth model containing 12 predictors with an adjusted $R$-squared of 0.9936 . Clearly the presence of dependent predictors creates unstable models. Compared to the model given in Equation (2), the increase of the adjusted $R$-squared is very small with regard to the increase of predictors in the model. It is therefore evident that these four models are outperformed and will not be retained.

Furthermore, we also performed linear regressions with, in addition, quadratic terms and also semi-logarithmic regressions. However the linear model outperformed these two extensions.

Finally, to avoid collinearity problems in a smarter way, we also conducted Principal Component Regressions (PCR). The results were not as good as the linear regression. This comes from the fact that the relevant variables explaining the price are, with the principal component analysis, diluted into many principal factors. These factors are computed to explain, in an optimal way, the variance of all predictors considered but they are not necessarily, as in our case, optimized to explain the variation of the price. For our case, if we handle collinearity by excluding variables, the standard linear regression outperforms PCR. However, to be fair, we have to mention that PCR outperforms standard regression when collinearity is not treated. We also recognize that in many cases PCR is clearly the best method to deal with collinearity problems.

In the proposed hedonic model, the car price increases, on average, by 259 Swiss francs when the power increases by 1 PS. The price decreases, on average, by 639 Swiss francs when the probability of having a car breakdown during the next year increases by one per thousand. We also see that if the car belongs to the superior category, its price increases, on average, by 8346 Swiss francs. This can be explained by two reasons. First, in this category, the quality of materials and the finishing touches are better than in other categories. Second, as we mentioned earlier, for each model, we only took into account the basic version satisfying some minimal criterion. In the superior category, the basic version includes features that are optional in other categories.

The retained hedonic model contains only three predictors. This was possible since, for each car model, we only took into consideration the basic version with the minimal options described in Table 2. This homogeneity of the cars considered permitted us to avoid a car's optional features that were unnecessary for 
this research. More than that, these unnecessary data would have overwhelmed the regression and would have led to a less good hedonic model. The price of a car obviously depends on options like metallic paint, leather seats, sunroof, alloy wheels, automatic transmission, etc., that were not considered in this study.

In this study, we imposed that variables which are not strongly significant be discarded from the model. As mentioned earlier, we discarded predictors with a $p$-value above 0.01 . This selective choice is justified for this study, as the adjusted $R$-squared of the model is very high $(0.9797)$ but could be misleading for other cases.

\subsection{Main Findings and Discussion}

Using the hedonic model presented in Equation (2), we can now answer our initial question: "what are the prices of reliability, energy efficiency, and safety?". For the reliability label, we can assert the following proposition.

Proposition 1 In Switzerland, the price of a new car depends on the ADAC reliability label.

Indeed, we can confidently reject the hypothesis that the price does not depend on the reliability label (two sided $t$-test, $p$-value $=3.66 \cdot 10^{-6}$ ). This result is in line with comparable studies [15] [16]. Furthermore, the regression coefficient permits us to estimate the price of reliability. A decrease by one per thousand of the probability of having a car breakdown during the next year increases, on average, the car price by 639 Swiss francs.

For the energy efficiency label, the two sided $t$-test $p$-values lie between 0.8783 (A label) and 0.4647 (E label). These values are above all usual significance levels and we cannot reject the hypothesis that the price does not depend on the energy efficiency label. However, this result has to be taken with caution. Indeed, the energy label and the engine power are not independent, as a Kruskal-Wallis test ${ }^{2}$ on the variables POWER and ENERGY shows ( $p$-value $=0.0041)$. It is therefore not improbable that a possible effect of the variable ENERGY on the price was in fact taken into account in our model by means of the variable POWER. We reach here the limit of our quantitative model and have to admit that no definitive answer can be provided. This result has to be put into perspective with other studies obtaining mixed results [10] [18] [20] and studies showing the impact of energy efficiency on price [19] [21] [22]. A survey could be an alternative and indirect method to assess whether the price depends on the energy efficiency. However, such a study has to be carefully designed in order not to face a declarative bias. Using semi-structured interviews, [37] concludes that consumers do not take into account fuel economy when buying a car. This result is however contradicted by [38], where the authors used four quantitative surveys to establish that respondents' willingness to pay for fuel economy is, certainly, less than the potential savings but is consistent with the behavioural economic principle ${ }^{2} \mathrm{~A}$ Shapiro-Wilk test of normality with $p$-value $1.56 \cdot 10^{-6}$ shows that the normality assumption is not met for the variable POWER and the use of ANOVA is therefore not recommended. However, ANOVA would confirm the result ( $p$-value $=0.0003$ ). 
of loss aversion.

For the safety label, the two sided $t$-test $p$-values lie between 0.6117 (three stars) and 0.2705 (four stars). These values are above all the usual significance levels and we cannot reject the hypothesis that the price does not depend on the safety label. However, like the energy label, the safety label is not independent of the engine power, as a Kruskal-Wallis test ${ }^{3}$ on the variables POWER and SAFETY shows $\left(p\right.$-value $\left.=1.17 \cdot 10^{-4}\right)$. This result has to be taken, therefore, with extreme caution and, as with the energy efficiency label, we must admit that our question cannot be answered. Furthermore, studies using a similar hedonic model seem to contradict this result [15] [24] [25]. As with the energy efficiency, a survey could bring another interesting point of view on our problem. Using a quantitative questionnaire, [39] found that safety is the most important criterion in the purchase process of a new car. This result is confirmed by [40] where the authors found that safety and reliability are the two most important features that drivers consider when buying a car. Despite this network of incriminating proofs, our result could be, however, explained in the following way. Since 1997, when Euro NCAP was founded, their safety label became an important criterion guiding consumers' choices. So, all manufacturers made huge efforts to improve the safety of their cars. As a consequence, a decade later, many cars have been awarded five stars and it was even decided to tighten the label criteria in 2009. Following this trend to quality, we can observe that today a good safety label is a must in Switzerland. To illustrate this, five-star cars represent the huge majority of our sample (about 80\%) and cars that did not reach excellence are destined for niche markets. Indeed, almost all four-star cars belong to the micro-car class (some belong to the small-car class) and the worst rating is a good three stars for only two low cost cars. In light of this, it could be plausible that, though consumers attach great importance to safety, its effect on price is negligible since, except perhaps for niche markets, top safety is a must. Studies that contradict this hypothesis [15] [24] [25] were done in a period when car safety was not as high as today, namely prior to 2009 when Euro NCAP tightened the label criteria as a consequence of a generalised excellence in safety.

\subsection{The Price of Reliability versus Production Volume}

We found that reliability is an important salient attribute for cars and estimate the marginal price of reliability. As a subsidiary question, we would like to estimate if the price of reliability benefits from possible economies of scale. To do so, we will see the problem from the point of view of the manufacturer. The production costs related to reliability can be split into fixed costs and variable costs that depend on the production volume. Reasonably, we assume that variable costs are linear. We can write this as follows.

$$
C(\mathrm{~s})=C_{0}+C_{1} \cdot s
$$

${ }^{3}$ ANOVA was not used as the normality assumption is not met. However, ANOVA would confirm the result $(p$-value $=0.0045)$. 
where $C(s)$ represent the total costs for reliability, $C_{0}$ the fixed costs, $C_{1}$ the marginal costs and $s$ the quantity produced. Hence, the reliability costs per car are given by

$$
\frac{C(s)}{s}=\frac{C_{0}}{s}+C_{1} .
$$

In the hedonic model presented in Equation (2), the second term (i.e. $C_{1}$ ) was considered, whereas the first term (i.e. $C_{0} / s$ ), which represents the economies of scale, was not. We therefore extend the regression model to also incorporate this first term as a new predictor. Data for production volumes were obtained from [41]. For this new regression, the two sided $t$-test $p$-value associated with our new factor is 0.2038 . The result is clear: this new predictor is not statistically significant and has to be discarded from the regression. We can thus conclude that the price of reliability does not incorporate possible economies of scale.

\section{Conclusions}

In this study, we investigated, using a hedonic approach, whether the price of new cars in Switzerland depends on three popular quality labels and established the following results.

- The price depends on the ADAC reliability label. More precisely, a decrease by one per thousand of the probability of having a breakdown during the next year increases, on average, the car price by 639 Swiss francs.

- Due to collinearity issues, we cannot establish whether the SFOE energy label has an impact on price.

- Due to collinearity issues, we cannot establish whether the Euro NCAP safety label has an impact on price.

These results illustrate superbly the strengths and limitations of hedonic pricing. When used with proper judgement, hedonic regression can be a powerful tool to estimate the salient attributes value of a good or a service. However, before making any conclusion, the hedonic model has to be analysed with a critical point of view. Special attention should be paid to collinearity and to the predictors retained in or discarded from the model. For example, in this study, out of three questions, we were able to answer only one.

The research proposed in this article is original in two ways. To our knowledge, it is the first time that the ADAC reliability label has been used in a hedonic pricing model. We were able to show that this popular label in Switzerland has an impact on the car price. This confirms results from other studies which showed that reliability is an important salient attribute. The second original contribution is the choice of the sample. Indeed, to avoid overwhelming the regression with many options, we only took into consideration car models with homogeneous optional features. Actually, for each car model, only the basic model with some minimal options was considered. We were surprised not to find other studies concerning cars following the same method, as it leads to a 
model with strong explanation and few predictors.

For further research, it would be interesting to tackle the problem with conjoint analysis, another method that permits estimating the value of salient attributes [42] [43]. Unlike hedonic pricing, which deals with revealed preferences, conjoint analysis relies on stated preferences. This technique has been already used for cars (see for example [44] [45]), but not with the same attributes as the present study.

\section{Acknowledgements}

This work was supported by RCSO Economie et Management (HES-SO).

\section{Conflicts of Interest}

The author declares no conflicts of interest regarding the publication of this paper.

\section{References}

[1] ADAC (2015) ADAC Pannenstatistik 2015. https://www.adac.de/

[2] Consumers Reports (2016) Guide to Car Reliability. http://www.consumerreports.org/

[3] SFOE (2016) Energy Label. http://www.bfe.admin.ch/energieetikette/00886/

[4] Euro NCAP (2016) Safety Rating System. http://www.euroncap.com/

[5] Haas, G.C. (1922) Sale Prices as a Basis for Farmland Appraisal. Technical Bulletin, vol. 9, University of Minnesota, Agricultural Experiment Station.

[6] Court, A.T. (1939) Hedonic Price Indexes. The Dynamics of Automobile Demand, 99-119, General Motors Corporation.

[7] Griliches, Z. (1961) Hedonic Price Indexes for Automobiles: An Econometric of Quality Change. The Price Statistics of the Federal Government, 173-196, NBER.

[8] Lancaster, K.J. (1966) A New Approach to Consumer Theory. Journal of Political Economy, 74, 132-157. https://doi.org/10.1086/259131

[9] Rosen, S. (1974) Hedonic Prices and Implicit Markets: Product Differentiation in Pure Competition. Journal of Political Economy, 82, 34-55. https://doi.org/10.1086/260169

[10] Murray, J. and Sarantis, N. (1999) Price-Quality Relations and Hedonic Price Indexes for Cars in the United Kingdom. International Journal of the Economics of Business, 6, 5-27. https://doi.org/10.1080/13571519984287

[11] van Dalen, J. and Bode, B. (2004) Quality-Corrected Price Indices: The Case of the Dutch New Passenger Car Market, 1990-1999. Applied Economics, 36, 1169-1197. https://doi.org/10.1080/0003684042000247361

[12] Requena-Silvente, F. and Walker, J. (2006) Calculating Hedonic Price Indices with Unobserved Product Attributes: An Application to the UK Car Market. Economica, 73, 509-532. https://doi.org/10.1111/j.1468-0335.2006.00504.x

[13] Reis, H.J. and Santos Silva, J.M. (2006) Hedonic Prices Indexes for New Passenger Cars in Portugal (1997-2001). Economic Modelling, 23, 890-908. https://doi.org/10.1016/j.econmod.2006.04.003

[14] Matas, A. and Raymond, J.-L. (2009) Hedonic Prices for Cars: An Application to the 
Spanish Car Market, 1981-2005. Applied Economics, 41, 2887-2904. https://doi.org/10.1080/00036840701720945

[15] Carleton Asher, C. (1992) Hedonic Analysis of Reliability and Safety for New Automobile. The Journal of Consumer Affairs, 26, 377-396. https://doi.org/10.1111/j.1745-6606.1992.tb00033.x

[16] Betts, S.C. and Taran, Z. (2006) A Test of Prospect Theory in the Used Car Market: The Non-Linear Effects of Age and Reliability on Price. Academy of Marketing Studies Journal, 10, 57-75.

[17] Prieto, M., Caemmerer, B. and Baltas, G. (2015) Using a Hedonic Price Model to Test Prospect Theory Assertions: The Asymmetrical and Nonlinear Effect of Reliability on Used Car Prices. Journal of Retailing and Consumer Services, 22, 206-212. https://doi.org/10.1016/j.jretconser.2014.08.013

[18] Goodman, A.C. (1983) Willingness to Pay for Car Efficiency: A Hedonic Price Approach. Journal of Transport Economics and Policy, 17, 247-266.

[19] Atkinson, S.E. and Halvorsen, R. (1984) A New Hedonic Technique for Estimating Attribute Demand: An Application to the Demand for Automobile Fuel Efficiency. The Review of Economics and Statistics, 66, 417-426. https://doi.org/10.2307/1924997

[20] Witt, R. (1997) The Demand for Car Fuel Efficiency: Some Evidence for the UK. Applied Economics, 29, 1249-1254. https://doi.org/10.1080/00036849700000015

[21] Espey, M. and Nair, S. (2005) Automobile Fuel Economy: What Is Worth? Contemporary Economic Policy, 23, 317-323. https://doi.org/10.1093/cep/byi024

[22] Alberini, A., Bareit, M. and Filippini, M. (2016) What Is the Effect of Fuel Efficiency Information on Car Prices? Evidence from Switzerland. The Energy Journal, 37, 315-342. https://doi.org/10.5547/01956574.37.3.aalb

[23] Hogarty, T.F. (1975) Price-Quality Relations for Automobiles: A New Approach. Applied Economics, 7, 41-51. https://doi.org/10.1080/00036847500000006

[24] Dreyfus, M.K. and Viscusi, W.K. (1995) Rates of Time Preference and Consumer Valuations of Automobile Safety and Fuel Efficiency. Journal of Law and Economics, 38, 79-105. https://doi.org/10.1086/467326

[25] Andersson, H. (2005) The Value of Safety as Revealed in the Swedish Car Market: An Application of the Hedonic Pricing Approach. Journal of Risk and Uncertainty, 30, 211-239. https://doi.org/10.1007/s11166-005-1154-1

[26] Akaike, H. (1973) Information Theory and an Extension of the Maximum Likelihood Principle. 2nd International Symposium on Information Theory, Tsahkadsor, 2-8 September 1973, 267-281.

[27] Akaike, H. (1974) A New Look at the Statistical Model Identification. IEEE Transactions on Automatic Control, 19, 716-723. https://doi.org/10.1109/TAC.1974.1100705

[28] Miller, A.J. (1984) Selection of Subsets of Regression Variables. Journal of the Royal Statistical Society: Series A, 147, 389-425. https://doi.org/10.2307/2981576

[29] Hurvich, C.M. and Tsai, C.-L. (1990) The Impact of Model Selection on Inference in Linear Regression. The American Statistician, 44, 214-217.

[30] Derksen, S. and Keselman, H.J. (1992) Backward, Forward and Stepwise Automated Subset Selection Algorithms: Frequency of Obtaining Authentic and Noise Variables. British Journal of Mathematical and Statistical Psychology, 45, 265-282. https://doi.org/10.1111/j.2044-8317.1992.tb00992.x

[31] Farrar, D.E. and Glauber, R.R. (1967) Multicollinearity in Regression Analysis: The 
Problem Revisited. The Review of Economics and Statistics, 49, 92-107. https://doi.org/10.2307/1937887

[32] Gordon, R.A. (1968) Issues in Multiple Regression. American Journal of Sociology, 73, 592-616. https://doi.org/10.1086/224533

[33] Triplett, J.E. (1969) Automobiles and Hedonic Quality Measurement. Journal of Political Economy, 77, 408-417. https://doi.org/10.1086/259524

[34] Pearson, K. (1901) On Lines and Planes of Closest Fit to Systems of Points in Space. Philosophical Magazine, 2, 559-572. https://doi.org/10.1080/14786440109462720

[35] Hotelling, H. (1933) Analysis of a Complex of Statistical Variables into Principal Components. Journal of Educational Psychology, 24, 417-441. https://doi.org/10.1037/h0071325

[36] TCS (2016) Recherche Auto TCS. https://www.tcs.ch/

[37] Turrentine, T.S. and Kurani, K.S. (2007) Car Buyers and Fuel Economy? Energy Policy, 35, 1213-1223. https://doi.org/10.1016/j.enpol.2006.03.005

[38] Greene, D.L., Evans, D.H. and Hiestand, J. (2013) Survey Evidence on the Willingness of US Consumers to Pay for Automotive Fuel Economy. Energy Policy, 61, 1539-1550. https://doi.org/10.1016/j.enpol.2013.05.050

[39] Koppel, S., Charlton, J., Fildes, B. and Fitzharris, M. (2008) How Important Is Vehicle Safety in the New Vehicle Purchase Process? Accident Analysis and Prevention, 40, 994-1004. https://doi.org/10.1016/j.aap.2007.11.006

[40] Vrkljan, B.H. and Anaby, D. (2011) What Vehicle Features Are Considered Important When Buying an Automobile? An Examination of Driver Preferences by Age and Gender. Journal of Safety Research, 42, 61-65.

https://doi.org/10.1016/j.jsr.2010.11.006

[41] Left-Lane (2015) European Car Sales Data. http://left-lane.com/

[42] Green, P.E. and Rao, V.R. (1971) Conjoint Measurement for Quantifying Judgmental Data. Journal of Marketing Research, 8, 355-363.

[43] Green, P.E. and Srinivasan, V. (1978) Conjoint Analysis in Consumer Research: Issues and Outlook. Journal of Consumer Research, 5, 103-123. https://doi.org/10.1086/208721

[44] Wu, W.Y., Liao, Y.K. and Chatwuthikrai, A. (2014) Applying Conjoint Analysis to Evaluate Consumer Preferences toward Subcompact Cars. Expert Systems with Applications, 41, 2782-2792. https://doi.org/10.1016/j.eswa.2013.10.011

[45] Chowdhury, M., Salam, K. and Tay, R. (2016) Consumer Preferences and Policy Implications for the Green Car Market. Marketing Intelligence \& Planning, 34, 810-827. https://doi.org/10.1108/MIP-08-2015-0167 